\title{
Effect of streptozotocin induced diabetes on rat hippocampus
}

\author{
Pamidi N, Satheesha Nayak B \\ Department of Anatomy, Melaka Manipal Medical College, Manipal, Karnataka, India. \\ narendra.pamidi@gmail.com
}

\begin{abstract}
Objective: The present study was designed to evaluate the diabetes induced neuronal damage in the hippocampus of the rat brain.

Background: Diabetes mellitus is an endocrine metabolic disorder of impaired carbohydrate, fat and protein metabolisms characterized by chronic hyperglycemia. The neurological consequences of diabetes mellitus in the brain have gained attention most recently.

Materials and methods: Male albino rats of Wistar strain, aged 30 days were used. The rats were divided into (A) Normal Control (B) Vehicle Control (C) 15 days of Streptozotocin (STZ), (D) 30 days of STZ, (E) 45 days of STZ, (F) 60 days of STZ diabetic groups ( $n=6$ in each group). Blood glucose levels and body weight were measured before STZ injection, 2 days after STZ injection and on the day of sacrifice. At the end of the experimental period rats were scarified and brains were processed for cresyl violet staining and the number of survived neurons in the hippocampus was quantified.

Results: Microscopic examination of cresyl violet stained sections of diabetic rat hippocampus showed significant and reliable changes. There was a significant difference in the number of survived neurons especially in 30 days of STZ, 45 days of STZ and 60 days of STZ diabetic groups compared to normal control group.

Conclusion: The results of our study indicated that diabetic complications can cause rapid damage to the neurons in the hippocampus (Fig. 12, Ref. 22). Full Text in PDF www.elis.sk.

Key words: streptozotocin, diabetes, hippocampus, neurons.
\end{abstract}

Diabetes mellitus is a metabolic syndrome of impaired carbohydrate, fat and protein metabolisms, caused by either lack of insulin secretion or decreased sensitivity of the tissues to insulin, characterized by chronic hyperglycemia. It is associated with chronic complications such as nephropathy, angiopathy, retinopathy and peripheral neuropathy (Biessels et al, 1994). Many organ systems are affected by diabetes, including the brain, which undergoes changes that may increase the risk of cognitive decline. The effects of diabetes mellitus on brain complications are increasingly recognized (Revsin et al, 2005).

The prevalence of cognitive dysfunction, depression, and anxiety is about 2-fold higher in diabetic patients than in the general population. Miyata et al (2006) have reported that STZ induced diabetic rodents showed cognitive impairment, depressive and anxiety behavior. Brain damage following cerebral ischemia/reperfusion was worsened distinctly under diabetic conditions (Wang et al, 2001). Type 1 diabetes mellitus results in brain disturbances like hypersensitivity to stress, cognitive disturbance, increased risk of stroke and dementia. A STZ model of type 1 diabetes, characterized by low insulin and high plasma glucose levels, affects not only body's overall metabolism but also brain activity (Zhang et

Department of Anatomy, Melaka Manipal Medical College, Manipal, Karnataka, India

Address for correspondence: N. Pamidi, PhD, Dept of Anatomy, Melaka Manipal Medical College (Manipal Campus), International Centre for Health Sciences, Manipal-576104, Karnataka, India.

Phone: +91820.2922642, Fax: +91820.2571905 al, 2008). Ryan has reported that learning and memory capacities are reduced in type 1 diabetes mellitus patients (Ryan, 1988).

The hippocampus plays a key role in learning and memory activities (Bliss et al, 1993). Hippocampus is the centre for alterations associated with diabetes in the central nervous system (Saravia et al, 2004). In a study by Stranahan et al (2008) rats treated with STZ showed hyperglycemia with increased corticosterone and impairments of hippocampal synaptic plasticity and learning. STZ induced diabetic rats showed learning deficits and impaired long-term potentiation in the CA1-field of the hippocampus (Kamal et al, 1999). In a study by Joghataie et al (2007) diabetes induced a reduction in the spine density of apical dendrites of medial prefrontal cortex neurons only in two-month diabetic rats. Recent studies revealed that STZ induced diabetes significantly reduced the number of proliferating cells in the dentate gyrus of rats by altering the hippocampal synaptic plasticity (Kamal et al, 2000, Jackson-Guilford et al, 2000). In this connection in the present study, we studied the amount of damage to the hippocampal neurons using cresyl violet staining in STZ induced experimental diabetes.

\section{Materials and methods}

Male albino rats of Wistar strain, aged 30 days, weighing 65-85 $\mathrm{g}$ were used. All animals were maintained under 12:12 hour dark: light environment, in institutional animal house. Animals were housed in polypropylene cages; paddy husk was used for bedding. Animals were given water ad libitum, the standard pellets 
(Hindustan lever) were used for feeding. Research was conducted in compliance with policies and principles contained in the institutional animal ethical committee guidelines. The experimental protocol was approved by the institutional animal ethical committee for experimental clearance (IAEC/KMC/07/2007-2008). Animals were maintained in accordance with the guidelines of government of India for use of laboratory animals.

\section{Animal groups}

The rats were divided into (A) Normal Control (NC) (B) Vehicle Control (VC) (C) 15 days of STZ, (D) 30 days of STZ, (E) 45 days of STZ, (F) 60 days of STZ diabetic groups ( $n=6$ in each group). Rats in NC group were remained undisturbed in their home cage and rats in VC group were fed with vehicle solution (Citric acid buffer, $\mathrm{pH} 4.5$ ). Diabetes was induced in diabetic group of rats by a single intraperitoneal injection of STZ, and sacrificed after 15, 30, 45 and 60 days of diabetic period.

\section{Preparation STZ solution}

Fresh STZ solution was prepared in $0.1 \mathrm{M}$ citrate buffer $(\mathrm{pH}$ 4.5). STZ solution of $10 \mathrm{mg} / \mathrm{ml}$ was prepared by dissolving STZ in ice-cold citrate buffer. This is to prevent decomposition of STZ by temperature. The solution was maintained in cold condition by keeping it in a tray of ice cubes.

\section{Induction of experimental diabetes}

Rats were fasted the night before STZ drug administration. A single intraperitoneal injection of STZ (Sigma, St, Louis Mo., USA) dissolved in citrate buffer $(0.1 \mathrm{M}, \mathrm{pH} 4.5)$ was delivered within five minutes at a dose of $40 \mathrm{mg} / \mathrm{kg}$ body weight. The animals were allowed to drink 5\% glucose solution to overcome druginduced hypoglycemia. Vehicle Control animals were injected with the vehicle (Citrate buffer, $\mathrm{pH} 4.5$ ).

\section{Blood glucose and body weight measurement}

Blood was collected from orbital plexus and tail vein of rats and blood glucose levels of rats were measured before STZ injection, 2 days after STZ injection and on the day of sacrifice. Blood sugar was estimated with the 'Accu-Chek Advantage Glucose Monitor'. $60-130 \mathrm{mg} / \mathrm{dl}$ is the range of normal blood glucose level of rats. Rats having blood glucose levels above $225 \mathrm{mg} / \mathrm{dl}$ after the injection of STZ were considered as diabetic and were used for the experiment. Body weights were measured before STZ injection, 2 days after STZ injection and on the day of sacrifice.

\section{Histological study of hippocampus}

Tissue fixation and processing for paraffin sectioning

Rats were deeply anesthetized with anesthetic ether, heart was exposed by opening the rib cage. About $100 \mathrm{ml}$ of the heparinized saline was pumped in to the left ventricle through a cannula and right atrium was cut open for the outflow of saline and blood. After saline pumping, transcardial perfusion of $300 \mathrm{ml}$ of $4 \%$ paraformaldehyde (Prepared in $0.1 \mathrm{M}$ citrate buffer) was done. The brains were removed and were post fixed for 48 hours in the Para formaldehyde. The brains were processed through ascending grades of alcohol $50 \% 24 \mathrm{hrs}, 70 \% 24 \mathrm{hrs}, 90 \% 12 \mathrm{hrs}, 100 \% 12$ hrs). Clearing of alcohol was done by immersing the brain tissues in xylene for about 1-2 hours. After clearing in xylene paraffin blocks were made in an embedding bath.

\section{Sectioning the tissues}

Coronal sections of $5 \mu \mathrm{m}$ thickness were cut from the dorsal hippocampus using a rotary microtome (Leica RM 2245, Germany). Twenty five to thirty sections from each animal were mounted serially on air dried gelatinized slides. The sections were stained using $0.01 \%$ cresyl violet stain and sections were dehydrated, mounted with DPX.

\section{Cresyl violet staining}

Gelatinized slides having brain sections were immersed in xylene solution for 15 minutes and then slides were immersed in ascending grades of alcohols in the following order, absolute alcohol - 1 minute, $90 \%$ alcohol - 2 minutes, $70 \%$ alcohol - 2 minutes, $50 \%$ alcohol -2 minutes. After processing through alcohol, slides were immersed in distilled water for 10 minutes and were stained for 25-30 minutes at temperature $60^{\circ}$ in $0.1 \%$ cresyl violet stain and then allowed to cool at room temperature. Stained sections were again immersed in distilled water for 5 minutes and in ascending grades of alcohols ( $70 \%, 90 \%$ ) for 2 minutes. Finally, sections were dipped in xylene for clearing and mounted with DPX.

\section{Cell counting}

Quantification of neurons in the sub regions of hippocampus (CA1, CA2, CA3, dentate hilus, dentate gyrus) was done using a light microscope under 40X (Magnus, Olympus Pvt. Ltd. New Delhi, India). To avoid manual bias slides from different groups were coded while counting. Ten sections from each rat were considered. The numbers of viable neurons (Neurons with distinct nucleus) were counted. Cells with darkly stained shrunken cell body and cells with fragmented nuclei were excluded from quantification.

\section{Data analysis}

Data was analyzed using analysis of variance (ANOVA) with Bonferroni's multiple comparison test as post hoc test (Graph pad prism 2.01 software). Values are expressed as mean \pm standard error of mean (SEM). A probability level of $p<0.05$ was used as the criterion for statistical significance.

\section{Results}

Blood glucose levels

There was no significant difference in the blood glucose levels of $\mathrm{NC}$ and $\mathrm{VC}$ group rats and the levels were stable throughout the experimental period. The blood glucose levels were as low as in $\mathrm{NC}$ and $\mathrm{VC}$ groups in the diabetic groups at the beginning of the experiment (before injection of STZ) (NC: $94.5 \pm 4.07, \mathrm{VC}$ : $92.83 \pm 3.06,15$ days of STZ: $94.5 \pm 3.442,30$ days of STZ: 92.83 \pm 3.038 , 45 days of STZ: $94.67 \pm 4.216,60$ days of STZ: 93.33 $\pm 4.573)$. All the rats in the diabetic groups became diabetic after 2 days following the STZ injection. The blood glucose levels of 


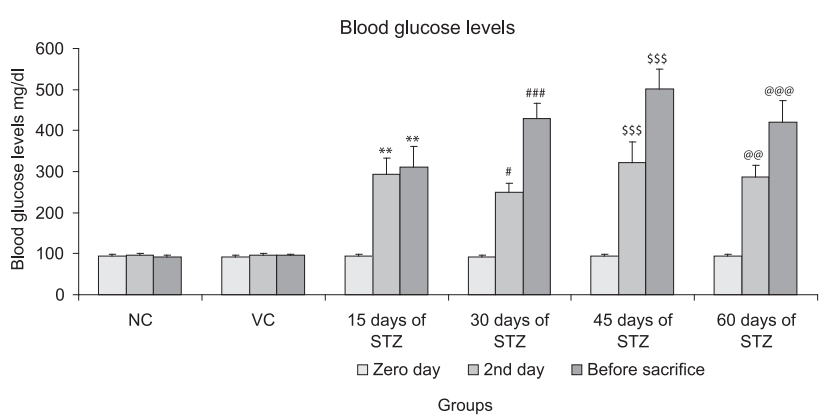

Fig. 1. Bar graph showing the blood glucose levels. Bars represent mean + standard error of mean. NC vs 15 days of STZ: ** p $<0.01$, NC vs 30 days of STZ: ${ }^{\#}<0.05,{ }^{\#} p<0.001$, NC vs 45 days of STZ: ${ }^{\text {sss }} p<$ 0.001, NC vs 60 days of STZ: ${ }^{\circledR @} p<0.01$, ${ }^{\circledR @}$ p $<0.001$.

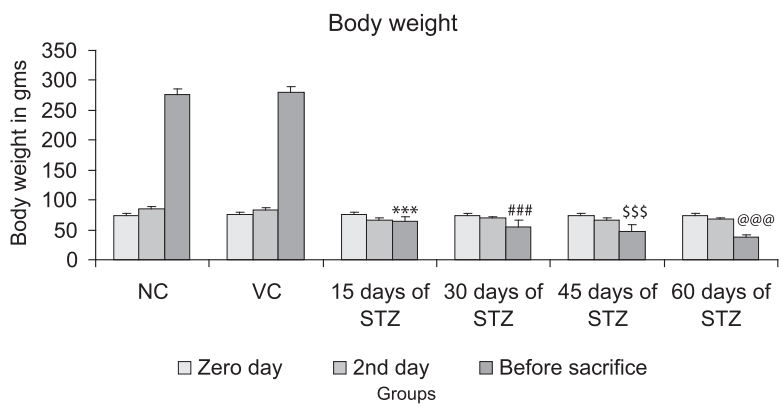

Fig. 2. Bar graph showing the body weight. Bars represent mean + standard error of mean. NC vs 15 days of STZ: *** $p<0.001$, NC vs 30 days of STZ: \# ${ }^{\prime}<0.001$, NC vs 45 days of STZ: ${ }^{\text {ss }} p<0.001$, NC vs 60 days of STZ: ${ }^{\circledR @} \mathrm{p}<0.001$.

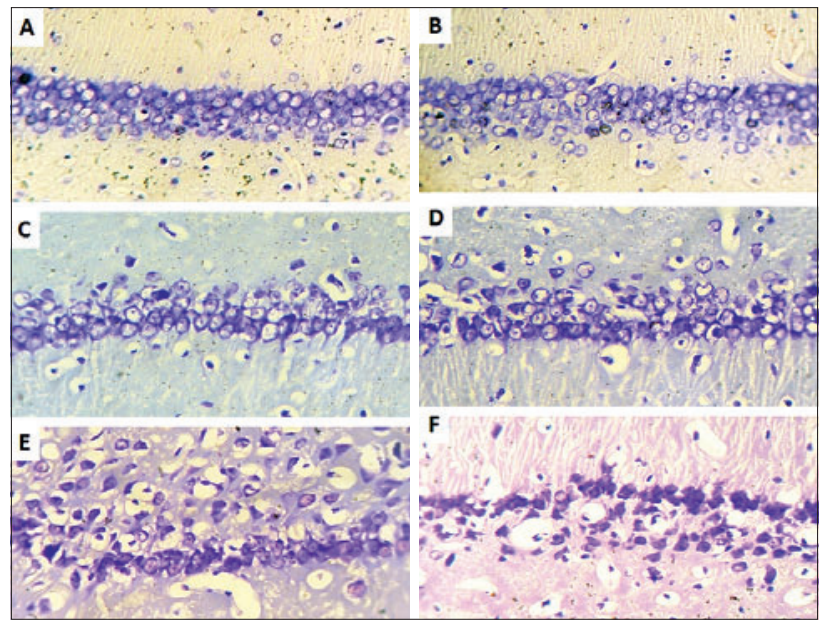

Fig. 3. Cresyl violet stained photomicrographs (A-NC, B-VC, C-15 days of STZ, D-30 days of STZ, E-45 days of STZ, F-60 days of STZ) showing the effect of STZ induced diabetes on the number of surviving neurons in the CA1 region of hippocampus in different groups. Significant decrease in the number of surviving neurons in $\mathbf{4 5}$ days of STZ and 60 days of STZ groups was observed.

diabetic rat groups were significantly increased in comparison to NC groups (NC: $96.33 \pm 3.58, \mathrm{VC}: 95.83 \pm 4.35,15$ days of STZ: $293.2 \pm 40.03(\mathrm{p}<0.01), 30$ days of STZ: $249.2 \pm 23.34(\mathrm{p}<$ $0.05), 45$ days of STZ: $322.3 \pm 50.74(\mathrm{p}<0.001), 60$ days of STZ: $286.5 \pm 28.34(\mathrm{p}<0.01))$ and remained hyperglycemic throughout

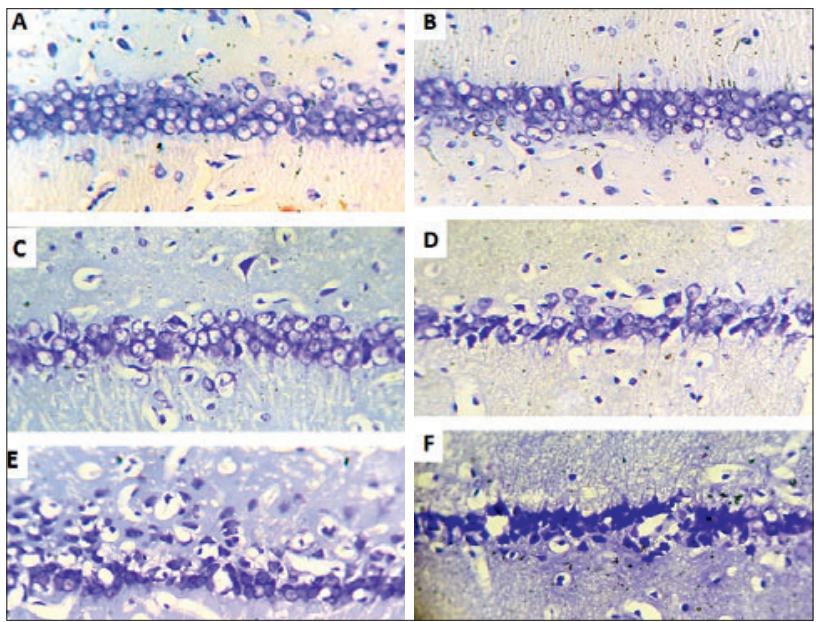

Fig. 4. Cresyl violet stained photomicrographs (A-NC, B-VC, C-15 days of STZ, D-30 days of STZ, E-45 days of STZ, F-60 days of STZ) showing the effect of STZ induced diabetes on the number of surviving neurons in the $\mathrm{CA2}$ region of hippocampus in different groups. Significant decrease in the number of surviving neurons in 45 days of STZ and 60 days of STZ groups was observed.

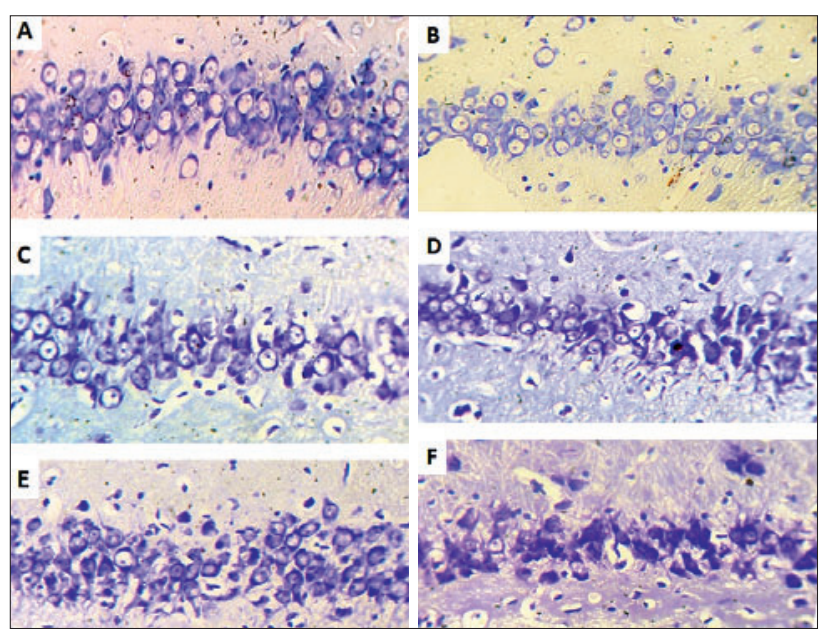

Fig. 5. Cresyl violet stained photomicrographs (A-NC, B-VC, C-15 days of STZ, D-30 days of STZ, E-45 days of STZ, F-60 days of STZ) showing the effect of STZ induced diabetes on the number of surviving neurons in the $\mathrm{CA3}$ region of hippocampus in different groups. Significant decrease in the number of surviving neurons in $\mathbf{3 0}$ days of STZ, 45 days of STZ and 60 days of STZ groups was observed.

the experimental period (blood glucose more than $250 \mathrm{mg} / \mathrm{dl}$ ). A highly significant increase in blood glucose levels was observed in diabetic groups when compared to the $\mathrm{NC}$ group before sacrifice (NC: $93 \pm 2.517, \mathrm{VC}: 96 \pm 3.08,15$ days of STZ: $311.8 \pm 50.11$ $(\mathrm{p}<0.01), 30$ days of STZ: $429.2 \pm 37.07$ ( $<<0.001), 45$ days of STZ: $501.7 \pm 47.15(\mathrm{p}<0.001), 60$ days of STZ: $421.3 \pm 52.08$ $(\mathrm{p}<0.001))$ (Fig. 1).

\section{Body weight}

The body weights of NC group and diabetic rats group did not differ significantly at the beginning of the experiment (NC: $74 \pm$ 4.17, VC: $76.83 \pm 3.44,15$ days of STZ: $75 \pm 4.09,30$ days of STZ: 


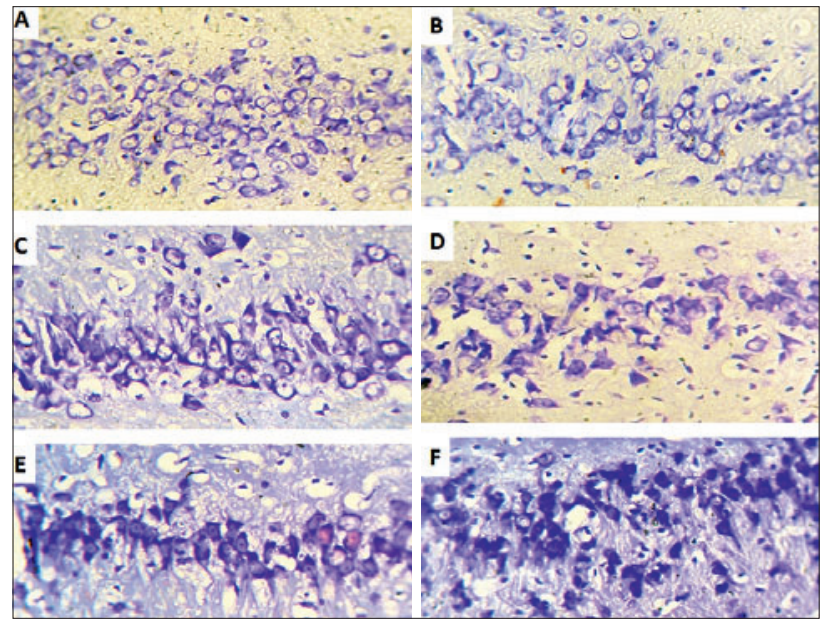

Fig. 6. Cresyl violet stained photomicrographs (A-NC, B-VC, C-15 days of STZ, D-30 days of STZ, E-45 days of STZ, F-60 days of STZ) showing the effect of STZ induced diabetes on the number of surviving neurons in the dentate hilus (DH) region of hippocampus in different groups. Significant decrease in number of surviving neurons in 15 days of STZ, 30 days of STZ 45 days of STZ and 60 days of STZ groups was observed.

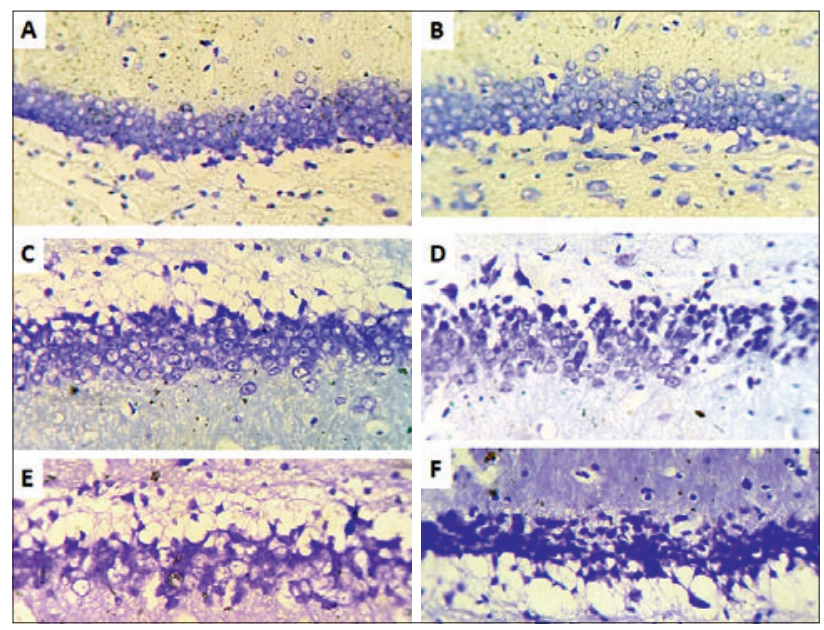

Fig. 7. Cresyl violet stained photomicrographs (A-NC, B-VC, C-15 days of STZ, D-30 days of STZ, E-45 days of STZ, F-60 days of STZ) showing the effect of STZ induced diabetes on the number of surviving neurons in the dentate gyrus (DG) region of different groups. Significant decrease in number of surviving neurons in 15 days of STZ, 30 days of STZ, 45 days of STZ and 60 days of STZ groups was observed.

$73.33 \pm 3.42,45$ days of STZ: $74.33 \pm 3.26,60$ days of STZ: 74.17 \pm 3.26 ). However, there was a highly significant reduction in the body weights of rats in the diabetic groups compared to the $\mathrm{NC}$ group of rats before sacrifice (NC: $276 \pm 9.5$, VC: $279.83 \pm 10.38$, 15 days of STZ: $65.17 \pm 7.55(\mathrm{p}<0.001), 30$ days of STZ: 55.33 $\pm 10.18(\mathrm{p}<0.001), 45$ days of STZ: $47.67 \pm 10.11(\mathrm{p}<0.001)$, 60 days of STZ: $38.17 \pm 3.84(\mathrm{p}<0.001)$ ) (Fig. 2).

Effect of STZ induced diabetes on the number of surviving neurons of hippocampus (Fig 3-12)

CA1 region of the hippocampus: Microscopic examination of cresyl violet stained sections of diabetic rat hippocampus showed

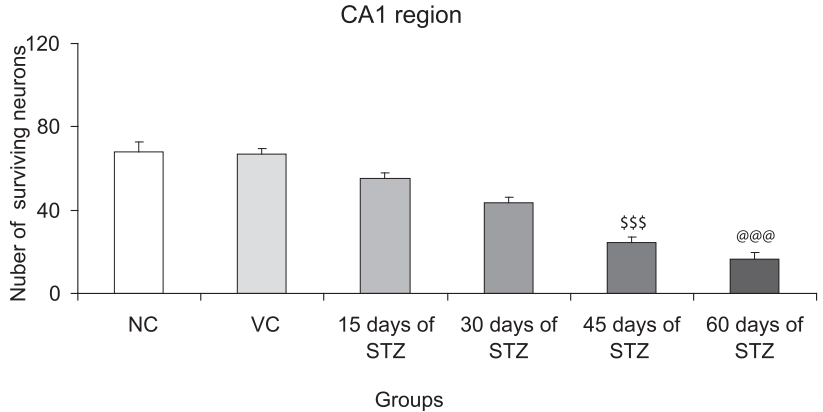

Fig. 8. Bar graph showing the number of survived neurons in the CA1 region of hippocampus. Bars represent mean \pm standard error of mean. NC vs 45 days of STZ: ${ }^{\text {ss }} p<0.001$, NC vs 60 days of STZ: (a) $\mathbf{p}<\mathbf{0 . 0 0 1}$.

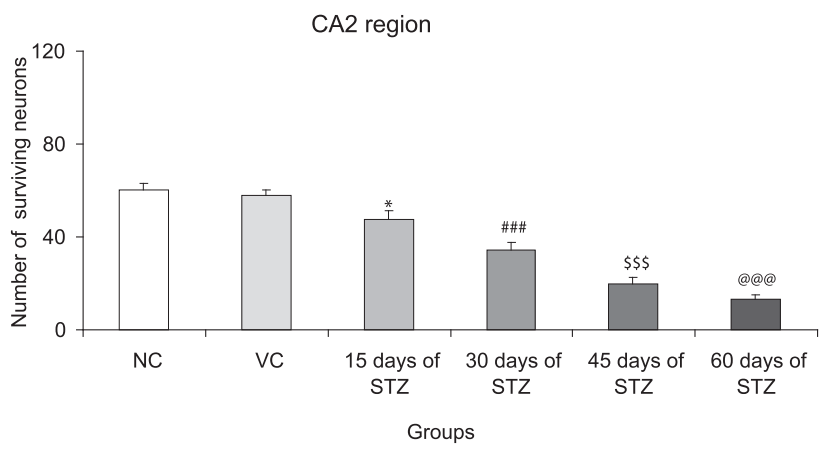

Fig. 9. Bar graph showing the number of survived neurons in the CA2 region of hippocampus. Bars represent mean \pm standard error of mean. NC vs 15 days of STZ: * $p<0.05$, NC vs 30 days of STZ: \#\# $p<0.001$, NC vs 45 days of STZ: ${ }^{\text {s\$s }} p<0.001$, NC vs 60 days of STZ: (a)@ $\mathbf{p}<\mathbf{0 . 0 0 1}$.

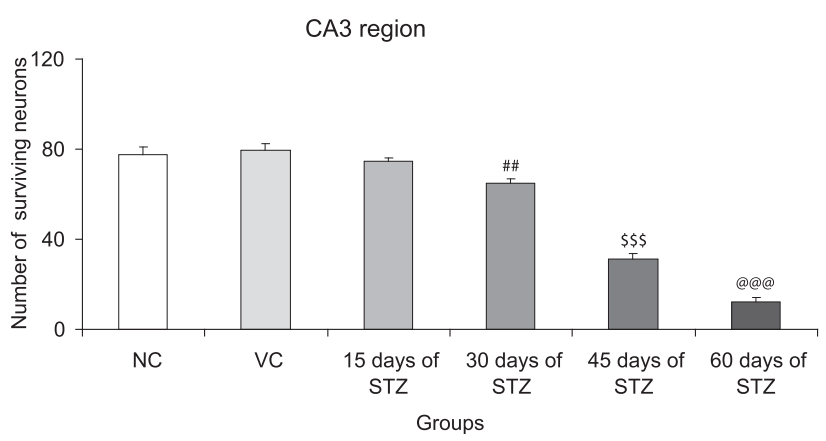

Fig. 10. Bar graph showing the number of survived neurons in the CA3 region of hippocampus. Bars represent mean \pm standard error of mean. NC vs 30 days of STZ: ${ }^{\#}<0.01, N C$ vs 45 days of STZ: ${ }^{\text {sSs }}$ $\mathrm{p}<\mathbf{0 . 0 0 1}$, NC vs 60 days of STZ: ${ }^{\circledR @ @ ~} \mathrm{p}<0.001$.

significant and reliable changes (NC: $68.17 \pm 4.52$, VC: $66.67 \pm$ 3, 15 days of STZ: $55.37 \pm 2.7,30$ days of STZ: $43.37 \pm 2.81,45$ days of STZ: $24.5 \pm 2.39$, 60 days of STZ: $16.67 \pm 3.15$ ). Counting the cresyl violet stained neurons of hippocampus showed a highly significant decrease in the number of surviving neurons in 45 days of STZ and 60 days of STZ groups $(p<0.001)$ in comparison to the $\mathrm{NC}$ group.

$C A 2$ region of the hippocampus: There was a significant decrease observed in the 15 days of STZ $(\mathrm{p}<0.05) 30$ days of STZ, 


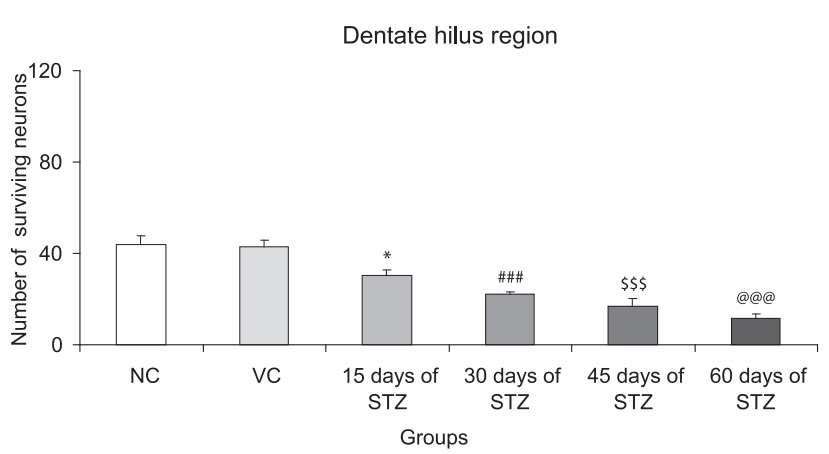

Fig. 11. Bar graph showing the number of survived neurons in the dentate hilus region of hippocampus. Bars represent mean \pm standard error of mean. NC vs 15 days of STZ: * $p<0.05$, NC vs 30 days of STZ: ${ }^{\# \#}$ p 0.001 , NC vs 45 days of STZ: ${ }^{\text {ss }} p<0.001$, NC vs 60 days of STZ: ${ }^{\circledR @} p<0.001$.

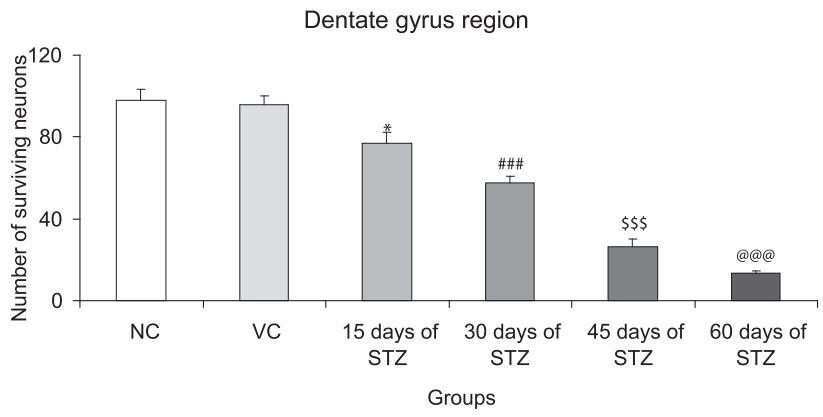

Fig. 12. Bar graph showing the number of survived neurons in the dentate gyrus region. Bars represent mean \pm standard error of mean. NC vs 15 days of STZ: * $p<0.05$, NC vs 30 days of STZ: vs 45 days of STZ: ${ }^{\text {ss }} p<0.001$, NV vs 60 days of STZ: ${ }^{\circledR @} p<0.001$.

45 days of STZ and 60 days of STZ ( $<<0.001)$ groups (NC: 60.33 $\pm 2.64, \mathrm{VC}: 57.67 \pm 2.39,15$ days of STZ: $47.67 \pm 3.93,30$ days of STZ: $34.5 \pm 3.12,45$ days of STZ: $20 \pm 2.46,60$ days of STZ: $13.17 \pm 2.04)$ in the number of surviving neurons in comparison to the $\mathrm{NC}$ group in the $\mathrm{CA} 2$ region of the hippocampus.

CA3 region of the hippocampus: Quantification of cells in CA3 region of hippocampus showed a significant decrease in the number of surviving neurons in Diabetic rats group (30 days of STZ $(\mathrm{p}<0.01), 45$ days of STZ and 60 days of STZ groups $(\mathrm{p}<0.001)$ compared to the NC group (NC: $7.33 \pm 3.78, \mathrm{VC}: 79.5 \pm 2.83,15$ days of STZ: $74.5 \pm 1.52,30$ days of STZ: $64.67 \pm 2.376,45$ days of STZ: $31.17 \pm 2.67,60$ days of STZ: $12.33 \pm 1.74$ ).

Dentate hilus region of the hippocampus: When compared to the NC group of rats, a significant decrease in number of surviving neurons in 15 days of STZ $(p<0.05), 30$ days of STZ 45 days of STZ and 60 days of STZ groups $(p<0.001)$ was observed in the Dentate hilus region of the hippocampus (NC: $44 \pm 3.52, \mathrm{VC}$ : $42.83 \pm 2.97,15$ days of STZ: $30.33 \pm 2.45,30$ days of STZ: $22 \pm$ 1.36, 45 days of STZ: $16.67 \pm 3.47,60$ days of STZ: $11.67 \pm 1.82$ ).

Dentate gyrus: Quantitative analysis of the neurons in the dentate gyrus showed a significant decrease in the number of surviving neurons in 15 days of STZ $(\mathrm{p}<0.05), 30$ days of STZ, 45 days of STZ and 60 days of STZ groups $(\mathrm{p}<0.001)$ when compared with the $\mathrm{NC}$ group (NC: $98 \pm 5.27, \mathrm{VC}: 96 \pm 4.07,15$ days of STZ: $77.17 \pm 4.94,30$ days of STZ: $57.33 \pm 3.24,45$ days of STZ: $26.33 \pm 3.56,60$ days of STZ: $13.33 \pm 1$. These results suggest that neuronal cell bodies are sensitive to stressful conditions such as diabetes.

\section{Discussion}

Diabetic neuropathy is one of the most common complications of diabetes. Severe as well as chronic hyperglycemia impairs the cerebral blood flow and affects the neural structure irreversibly. STZ induced diabetic model results in chronic oxidative stress. DM results in increased formation of free radicals by cellular glucose auto-oxidation with decreased antioxidant potential. In animals and in vitro models of diabetes, hyperglycemia along with the other complications of diabetes causes damage by activating the key pathways of glucose metabolism. Free radical causes cellular damage by initiating apoptosis in neurons and neuroglial cells. Oxidative stress in STZ induced diabetes might not be the only pathogenetic mechanism, other factors like impaired insulin signaling pathway may also be involved in development of the neuropathy (Dagon et al, 2007).

Neuronal dysfunctions and morphological changes in diabetic hippocampus may also be due to the reduced level of brain derive neurotrophic factor (Wisniewski et al, 2003). Severity and duration of diabetes bring alterations in the catecholaminergic system in an organized manner and also reduce the dopamine levels in the dopaminergic nigrostriatal system. Reduction in norepinephrine release was observed, in more than 30 days of diabetic duration (Gallego et al, 2003). STZ induced diabetic rats exhibited increased corticosterone levels (Stranahan et al, 2008). cAMP-response element binding (CREB) transcription factor is a component of intracellular signaling events that regulate a wide range of biological functions. CREB role has been well-documented in neuronal plasticity and long-term memory formation in the brain (Silva et al, 1998). Findings from Makimattila et al (2004) and Kumar et al (2010) revealed a significant down regulation of CREB in cerebral cortex, cerebellum, brain stem, hippocampus and hypothalamus and up regulation in corpus striatum of diabetic rats, when compared to controls.

In diabetic brain irregular influxes of glutamate or calcium can take place due to reduced expression of calcium binding proteins (Calbindin, Synaptophysin and Syntaxin). Neuronal excitation caused by glutamate and subsequent calcium influx into the cell may be the initiating factor for morphological alterations and neuronal cell death in diabetic conditions (Joghataie et al, 2007, Parihar et al, 2004). Both types of diabetes causes cerebral alterations by reducing hippocampal synaptic plasticity, abnormal hypothalamic neuropeptide expression, hippocampal astrogliosis, neurotoxicity and changes in glutamate neurotransmission increased circulating corticosterone levels, lack of periodicity, hypersensitivity to stress, and down-regulation of hippocampal glucocorticoid receptors may contribute to the damage. Diabetic patients can exhibit decreased memory and learning (Revsin et al, 2005).

In STZ induced diabetes the nerve damage is similar to nerve damage in human diabetic neuropathy [Reagan and McEwen, 
2002]. STZ results in decrease of insulin release by destructing the b-cells of the pancreas. Small amount of residual insulin is still present in the pancreas even after destruction of $b$ cells, and these diabetic animals can survive for several months but, the disease is fatal without treatment (Zhang et al, 2008). According to the existing data, diabetic effects on the central nervous system are delayed. Previous studies failed to find any changes in neuronal cell number using Nissl staining (Nitta et al, 2002).

Accordingly the present study was designed to further assess the damage inflicted to neurons in the hippocampus in experimental diabetes. Accountable histological alterations were seen in all the regions of hippocampus of diabetic rats when compared to the normal control group. The 30 days diabetic group showed significant changes in the histology of various hippocampal regions. In the hippocampus, neurons were shrunken and darkly stained with a small nucleus. All the sub regions of the hippocampus (CA1, CA2, CA3, dentate hilus, dentate gyrus) showed the most significant changes in 45 and 60 days of diabetes (Rats exposed to chronic diabetes). Our study proved that untreated diabetes mellitus can induce the damage in the central nervous system of the rat within two weeks of diabetic period or earlier. Our results showed that the number of survived neurons in the sub regions of the hippocampus (CA1, CA2, CA3, dentate hilus, dentate gyrus) of diabetic rat brain were decreased to about $75 \%$ in 15 days diabetic rats, $60 \%$ in 30 days diabetic rats, $35 \%$ in 45 days diabetic rats and it was $20 \%$ in 60 days diabetic rats compared to controls and prolonged untreated diabetes can lead to severe hippocampal neurodegeneration.

\section{Conclusions}

Results from our study indicated that diabetic complications can cause rapid damage to the neurons in the hippocampus of rat brain. The present data in the rat hippocampus may be useful to interpret the functional deficits of diabetic patients and better management of diabetes.

\section{References}

1. Biessels GJ, Kappelle AC, Bravenboer B, Erkelens DW, Gispen WH. Cerebral function in diabetes mellitus. Diabetologia 1994; 37 (7): 643-650.

2. Revsin Y, Saravia F, Roig P, Lima A, de Kloet ER, Homo-Delarche F, De Nicola AF. Neuronal and astroglial alterations in the hippocampus of a mouse model for type 1 diabetes. Brain Res 2005; 1038 (1): 22-31.

3. Miyata S, Saitoh A, Hirano S, Kamei J. Effect of streptozotocininduced diabetes on the function of the central nervous system in rodents. Nihon Shinkei Seishin Yakurigaku Zasshi 2006; 26 (2): 83-87.

4. Wang GX, Li GR, Wang YD, Yang TS, Ouyang YB. Characterization of neuronal cell death in normal and diabetic rats following experimental focal cerebral ischemia. Life Sci 2001; 69 (23): 2801-2810.

5. Zhang WJ, Tan YF, Yue JT, Vranic M, Wojtowicz JM. Impairment of hippocampal neurogenesis in streptozotocin-treated diabetic rats. Acta Neurol Scand 2008; 117 (3): 205-210.

6. Ryan CM. Neurobehavioral complications of type I diabetes. Examination of possible risk factors. Diabetes Care 1988; 11 (1): 86-93.
7. Bliss TV, Collingridge GL. Asynaptic model of memory: long-term potentiation in the hippocampus. Nature 1993; 361:31-39

8. Saravia F, Revsin Y, Lux-Lantos V, Beauquis J, Homo-Delarche F, De Nicola AF. Oestradiol restores cell proliferation in dentate gyrus and subventricular zone of streptozotocin-diabetic mice. J Neuroendocrinol 2004; 16 (8): 704-710.

9. Stranahan AM, Arumugam TV, Cutler RG, Lee K, Egan JM, Mattson MP. Diabetes impairs hippocampal function through glucocorticoid-mediated effects on new and mature neurons. Nat Neurosci 2008; 11 (3): 309-317.

10. Kamal A, Biessels GJ, Urban IJ, Gispen WH. Hippocampal synaptic plasticity in streptozotocin-diabetic rats: impairment of long-term potentiation and facilitation of long-term depression. Neuroscience 1999; 90 (3): 737-745.

11. Joghataie MT, Roghani M, Jalali MR, Baluchnejadmojarad T, Sharayeli M. Dendritic Spine Changes in Medial Prefrontal Cortex of Male Diabetic Rats Using Golgi-Impregnation Method. Arch Iranian Med 2007; 10 (1): 54-58.

12. Kamal A, Biessels GJ, Duis SEJ, Gispen W H. Learning and hippocampal synaptic plasticity in streptozotocin-induced diabetic rats:interaction of diabetes and ageing. Diabetologia 2000; 43 (4): 500-506.

13. Jackson-Guilford J, Leander JD, Nisenbaum LK. The effect of streptozotocin induced diabetes on cell proliferation in the rat dentate gyrus. Neurosci Lett 2000; 293 (2): 91-94.

14. Dagon Y, Avraham Y, Link G, Zolotarev O, Mechoulam R, Berry EM. The synthetic cannabinoid HU-210 attenuates neural damage in diabetic mice and hyperglycemic heochromocytoma PC12 cells. Neurobiol Dis 2007; 27 (2): 174-181.

15. Wisniewski K, Fedosiewicz-Wasiluk M, Holy ZZ, Car H, Grzeda E. Influence of NMDA, a potent agonist of glutamate receptors, on behavioral activity in 4-week streptozotocin-induced diabetic rats. Pol J Pharmacol 2003; 55 (3): 345-351.

16. Gallego M, Setien R, Izquierdo MJ, Casis O, Casis E. Diabetesinduced biochemical changes in central and peripheral catecholaminergic systems. Physiol Res 2003; 52 (6): 735-741.

17. Silva AJ, Kogan JH, Frankland PW, Kida S. CREB and memory. Annu Rev Neurosci 1998; 21:127-148.

18. Makimattila S, Malmberg-Ceder K, Hakkinen AM et al. Brain metabolic alterations in patients with type 1 diabetes-hyperglycemia-induced injury. J Cereb Blood Flow Metab 2004; 24 (12): 1393-1399.

19. Kumar TP, Antony S, Gireesh G, George N, Paulose CS. Curcumin modulates dopaminergic receptor, CREB and phospholipase $\mathrm{C}$ gene expression in the cerebral cortex and cerebellum of streptozotocin induced diabetic rats. J Biomed Sci 2010; 17:43.

20. Parihar MS, Chaudhary M, Shetty R, Hemnani T. Susceptibility of hippocampus and cerebral cortex to oxidative damage in streptozotocin treated mice: prevention by extracts of Withania somnifera and Aloe vera. J Clin Neurosci 2004; 11 (4): 397-402.

21. Reagan LP, McEwen BS. Diabetes, but not stress, reduces neuronal nitric oxide synthase expression in rat hippocampus: implications for hippocampal synaptic plasticity. Neuroreport 2002; 13 (14): 1801-1804.

22. Nitta A, Murai R, Suzuki N et al. Diabetic neuropathies in brain are induced by deficiency of BDNF. Neurotoxicol Teratol 2002; 24 (5): 695-701.

Received February 24, 2012. Accepted June 26, 2012. 